Recibido: 05-09-2016

Aceptado: 23-09-2016

\title{
La admisión de varones en los programas académicos de la Escuela de Posgrado de la UNIFÉ
}

\section{The admittance of men in the academic \\ programs Unife's Graduate School}

Agustín Campos Arenas, Ph. D.

RESUMEN. La admisión de varones a los Programas Académicos de Maestria y Doctorado de la Escuela de Posgrado de la UNIFÉ es materia de análisis en el presente artículo. El hecho se enfoca dentro del marco de la conversión de programas dirigidos a personas de un solo sexo a programas coeducacionales que sirve como antecedente a la decisión de la congregación del Sagrado Corazón de apertura a varones en la Escuela de Posgrado. Se presenta la situación en otras instituciones educativas de la Congregación con relación a la coeducación y la situación particular de la UNIFE al respecto, Se reconoce a los tres primeros varones que ingresaron a estudiar la maestría en educación y se recoge sus opiniones acerca de sus experiencias. Se concluye que esta situación, con los nuevos ingresos, es ya una grata realidad.

Palabras clave: Coedcuación, instituciones para estudiantes de un solo sexo, Universidad Femenina del Sagrado Corazón, escuela de posgrado.

Keywords: Coeducation, institutions for single-sex students, Universidad Femenina del Sagrado Corazón, postgraduate school.

Agustín Campos Arenas, Ph. D. UNIFE agustin@unife.edu.pe Programs of UNIFE's Postgraduate School is the subject of analysis in this article. The fact is focused within the framework of the conversion of programs directed to single-sex students to coeducational programs that serves as background of the decision of the Sacred Heart congregation to open the Graduate School to men. The situation regarding coeducation is present in other educational institutions of the Congregation and the particular situation of UNIFE in this regard. It recognizes the first three men who pursued the Master in Education and collects their opinions about their experiences. It is concluded that this situation, with the new admissions, is already a pleasant reality. 


\section{UNIFÉ-EPG}

\section{Introducción}

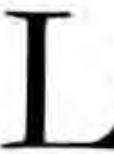

a historia universal muestra el desbalance entre la educación del hombre y el de la mujer. En las grandes civilizaciones, Grecia y Roma, se privilegiaba a la educación del hombre por cuestiones militares, económicas y politicas. Esto se mantuvo en las etapas posteriores de la historia. Es hasta el siglo XIX que comienza a plantearse mayores oportunidades educativas para las mujeres. La iglesia católica proclama la educación elemental (primaria) para los niños de todas las clases sociales y los protestantes consideran importante que a los niños y niñas se les deberían enseñar la biblia. Estas propuestas se extienden por varios países de Europa como Inglaterra, Escocia y Alemania. En estos países, así como en Estados Unidos (USA) se extiende la coeducación al universalizar el acceso de todos a la educación. Así en USA desde 1900 la mayoría de las escuelas de educación básica eran coeducacionales y a fines del siglo XX la mayoría de instituciones de educación superior que educaban a estudiantes de un mismo sexo se convirtieron en coeducacionales.

A manera de resumen Online Courses (2011) presenta una serie de conclusiones acerca de la educación segregada por sexo. Algunas de ellas son las siguientes:

- No se sabe a ciencia cierta si es beneficiosa.

- Actualmente existen instituciones educativas para estudiantes del mismo sexo.

- Los varones se benefician más que las mujeres.

- No preparan para la vida real en la coexisten los dos sexos.

- Las mujeres ganan mayor confianza en sus interrelaciones con los varones

- La educación superior es mayormente coeducacional para la cual no están preparados.

- Puede reforzar estereotipos: agresividad (hombres), sensualidad (mujeres).
A pesar de ello existen todavía instituciones para estudiantes del mismo sexo, las cuales se mantienen por la tradición y la calidad reconocida del servicio que ofrecen. Parmer (2013) considera que en un sistema coeducacional las mujeres están en desventaja y que la única manera de que la coeducación sea efectiva es cuando los "varones vean a sus compañeras como iguales".

Se trata, no solo permitir que ingresen a estudiar alumnos del otro sexo sino un cambio de percepción de lo que significa educar y coexistir.

\section{DESARROLLO}

El tema pedagógico inmerso en esta apertura es el de la coeducación, que según el diccionario de la lengua española proviene de coeducar que implica "enseñar en una misma aula y con un mismo sistema educativo a alumnos de uno y otro sexo" (DRAE). También es llamada "educación mixta" y definida como un sistema de educación donde estudian juntos hombres y mujeres.

\section{Razones}

Se consideró importante el derecho de las mujeres a tener igual educación que el hombre, lo cual no significa pérdida de identidad. Es igualdad en la oportunidad educativa en las mismas condiciones que los hombres. No es una mera "justificación" en la cual pueden estar en los mismos edificios y tener los mismos docentes, pero tener métodos o regímenes diferentes. Tampoco es una "coordinación", en la cual pueden estar en los mismos edificios, mismos docentes y misma administración, pero estar en escuelas separadas. Si no es una "educación idéntica" en todo sentido (Catholic Encyclopedia).

La propuesta de juntar a hombres y mujeres en un sistema educativo no ha escapado del entusiasmo y de la crítica. Por ejemplo, se menciona lo siguiente: 
Tabla 1

Consideraciones a favor y en contra acerca de la coeducación

\begin{tabular}{ll}
\hline A favor & En contra \\
\hline $\begin{array}{l}\text { - Hombres y mujeres estarán más preparados para } \\
\text { interactuar en el mundo real. }\end{array}$ & Menos preparados, nerviosos, incómodos \\
$\begin{array}{l}\text { - Las mujeres en instituciones femeninas están } \\
\text { afectadas en su desarrollo, pocas aspiraciones. }\end{array}$ & $\begin{array}{l}\text { Estudiantes más distrádos y las mujeres menos a participar en clase. } \\
\text { Seleccionan carreras tradicionales. }\end{array}$ \\
& $\begin{array}{l}\text { - En aulas de un solo sexo los estudiantes trabajan y } \\
\text { aprenden en el mismo nivel que sus compañeros, las } \\
\text { mujeres muestran más confianza. }\end{array}$ \\
\hline
\end{tabular}

La coeducación se mantiene en alto y ampliando la cobertura de la educación de los diferentes países. Debe indicarse que la coeducación recibió el apoyo de grandes educadores y organismos internacionales de educación en reconocimiento a las capacidades intelectuales y logros académicos de las mujeres (CQ Researcher, 1969).

\section{Desarrollo bistórico}

La coeducación ha devenido en una situación educativa estándar dese el siglo XIX; sin embargo, aún existen instituciones educativas dedicadas a estudiantes de un mismo sexo. En la mayoria de países se ha dado el desarrollo de ofrecer una educación mixta, pero aún hay restricción en los países de religión musulmana.

Es interesante anotar que intentos por dar educación mixta han existido mucho antes. Se menciona, en el nivel de Educación Básica, a la Dollar Academy como la primera institución escolar coeducacional en Escocia que inicia sus labores en 1818. En la educación superior se considera a Oberlin Collegiate Institute, de Oberlin-Ohio, como la primera institución coeducacional en Estados Unidos. Inició sus actividades en 1833 con 44 estudiantes: 29 hombres y 15 mujeres (College stats.org, 2015).

En Australiala primera escuela coeducacional fue Baker College y en China, en el intervalo 1910 - 1920, se crearon universidades femeninas (Ginling Women's University y Pekin Girls Higher Normal School). En 1920 la Universidad de Pekin permitió la asistencia de mujeres a clase; pero, sólo como oyentes. Las primeras instituciones coeducacionales de educación superior en China fueron Najing Higher Nomal Institute el cual se convirtió en la National Central University y luego en Nanjing University. Con motivo de la revolución en China se propuso que todas las instituciones educativas fueran coeducacionales.

En Francia la universidad de la Sorbona permitió el ingreso de mujeres en 1860. Asimismo, es obligatoria la coeducación desde 1957 para las escuelas de educación primaria y para las universidades desde 1975. En Hong Kong la primera institución coeducacional fue St. Paul's Coeducational College. En el Reino Unido, además de la Dollar Academy (Escocia), antes mencionada, se encuentra en Inglaterra la Bedales School que ofrece educación mixta desde 1898 y la University College London que ofrece educación superior desde 1878.

En Estados Unidos, las dos primeras instituciones de educación superior que ofrecian coeducación fueron Oberlin College en 1833. La primera universidad pública que deviene en coeducacional es la University of Iowa en 1855 y las primeras privada, Cornell University y University of Michigan, en 1870 . Una relación amplia de colleges y universidades se encuentra en la web.

Algunos de ellos se mencionan a continuación:

- Oberlin College, 1833

- Hillsdale College, 1844

- Kansas State University, 1863 
- Indiana University, 1867

- Boston University, 1869

- University of California, Berkeley, 1870

- Pensylvania State University, 1871

- Syracuse University, 1871

- Ohio State University, 1873

- Purdue University, 1875

- Florida State University, 1883

- Stanford University, 1891

- University of Chicago, 1892

- University of Pittsburgh, 1895

Un caso interesante es el de Florida State University. Habiéndose iniciado como institución coeducacional llamada Florida State College, en 1905 con motivo de la reorganización del sistema educativo del Estado de Florida se desdobla en dos instituciones: (1) The University of Florida, en Gainvesville, para hombres blancos y (2) Florida Female College, para mujeres. Para los estudiantes negros se crea una institución ad-hoc para ellos: Florida A\&M. Florida Female College cambia su nombre (1909) por el de Florida State College for Women, la que llegó a ser la tercera más grande del país. Después de la segunda guerra mundial, a los combatientes que retornaton al país (veteranos de guerra) se les dio facilidades para que realicen o reanuden sus estudios universitarios no realizados o interrumpidos por la guerra. El número fue grande, lo que hizo que el Gobernador del Estado en 1947 firmara el acta ordenando la conversión de esta institución a una coeducacional para recibir a estudiantes varones y cambió su nombre al de Florida State University con el cual se le conoce actualmente (Florida State University, About Florida State). Muchas otras instituciones, por efectos de los veteranos de guerra, permitieron el acceso a las instituciones femeninas a los varones.

Otro caso interesante es el de Princeton University que de ser siempre una universidad de varones, se transforma, en 1969, en una universidad coeducacional. Ese año se aceptaron 40 estudiantes mujeres para admisión y 90 por traslado. Igualmente, es el caso de Yale University, una universidad que durante 268 años era una universidad de varones, se convierten en coeducacional en 1969. Ese año 4300 mujeres postularon a esa universidad (CQ Researcher, 1969).

Recién a finales de 1970 las universidades de Oxford y Harvard comienzan a admitir mujeres (Hicks, 2004). Este proceso continúa actualmente. Asi, en pleno siglo XXI, continúan cambios hacia una educación mixta. Gill (2015) informa que una reconocida escuela tradicional privada de Australia, Armidale School, se convierte en una escuela coeduacional.

A pesar de todos los avances logrados en la coeducación, existe aún resistencia de ciertos sectores apelando a diferentes consideraciones. Por ejemplo, estableciendo diferencias cerebrales entre hombres y mujeres Michael Gurian (2001, en Wikipedia, 2016) púbica el libro Boys and Girls learn differently. Basado en esta premisa, se piensa que deben educarse por separado (Wikipedia, 2016).

Igualmente, en el caso de Princeton University, convertida a coeducacional, hay aún voces que cuestionan la decisión. Nancy Weiss Malkiels relata el proceso en su libro "Keep the Dammed Women Out: The Struggle for Coeducation" (Thomson Deveaux, 2016).

\section{De las instituciones educativas de la Congregación del Sagrado Corazón}

La educación, especialmente de la mujer, es de interés particular de las Religiosas del Sagrado Corazón de la Provincia del Perú. Para ello, promueven una serie de obras educativas en diferentes niveles del sistema educativo peruano. La congregación ha vivido la experiencia del cambio a la coeducación en algunas de sus instituciones educativas.

La Hna. Lastenia Fernández Maldonado (2016) manifiesta que:

- En el Instituto Pedagógico Nacional de Monterrico (IPNM) se inicia la coeducación en 1977, con motivo de la reforma educativa del gobierno militar convirtiéndose en la ESEP 
"Santa Magdalena Sofía Barat". Oficialmente regresa a la denominación de IPNM y se inicia la admisión de varones en el año 1991.

- El Colegio anexo al IPNM es sólo de mujeres en el periodo 1963 - 1982 y se convierte en mixto en el periodo de 1983 - 1991. Sin embargo, vuelve a funcionar solo para mujeres de 1992 - 1998, regresando, otra vez, a ser mixto de 1999 hasta la actualidad.

- En el Colegio Parroquial Madre Admirable del Agustino se comienza con la coeducación con la Reforma Educativa el gobierno militar con Educación Primaria. En Educación Secundaria se inicia en 1986.

- En el colegio Sagrado Corazón de Jaén se inicia la coeducación en 1994.

- En el colegio Sagrado Corazón Sophianum empieza en el año 2008

- Hay tres instituciones educativas que se mantienen con atención solo para mujeres: Chalet, Trujillo y la Universidad Femenina del Sagrado Corazón - UNIFE (Pregrado).

\section{Universidad Femenina del Sagrado Corazón}

La UNIFÉ fue creada por D.S. No 71 del 24 de diciembre de 1962 y comienza a funcionar el año 1963. En su finalidad y carácter señala se indica la formación integral de la mujer. En este enunciado está claro el servicio a la joven mujer peruana. Por más de 50 años, 53 exactamente, mantiene su centro formativo en la mujer. No obstante, el interés por la coeducación no ha estado ajeno en la Congregación del Sagrado Corazón como entidad fundadora y promotora de la universidad y de su representante, la hermana rectora.

\section{Antecedentes}

- En los años 70, con motivo del gobierno militar y de una nueva Ley Universitaria se debía cambiar autoridades en todas las universidades el país. Así, se elegió a la Hna. Raquel Corrales Gatjens como rectora de la UNIFÉ. Luego se designó al Dr. José Luis Bravo Amezaga como Vicerrector, siendo no solo laico sino varón. Se consideraba que el cargo debería ser desempeñado por una religiosa: sin embargo, por cuestiones coyunturas externas e internas de la UNIFÉ el Dr. Bravo ejerció el cargo por un tiempo.

- En los primeros años de la década de los 90, la Hna. Rectora de ese entonces Dra. Gladys Buzzio Zamora planteó al Consejo Universitario algunos cambios sustantivos en la UNIFÉ. Estos fueron:

- Que los cargos de Rector(a) y Vicerrector(a) pudieran ser ejercidos por laicos docentes de la universidad.

- Que pudieran acceder varones a los estudios de las carreras de la UNIFE incluyendo Postgrado.

Esto generó entusiasmo y dudas entre los miembros del Consejo y cuerpo docente. Después de las consultas a especialistas, asesores y a la Congregación, la Hna. Provincial se dirigió al Consejo universitario dando su consentimiento para que el cargo de Vicerrector (Académico y Administrativo) pueda ser ejercido por laicos. Las otras propuestas no se consideraban viables, en ese momento. Se hicieron los cambios en el Estatuto y en 1996 se eligieron a los primeros vicerrectores: Dr. Agustín Campos Arenas (Académico) y Dra. Carmen Cornejo Tavella (Administrativo). En este caso, también un varón ocupaba un cargo directivo.

Esta voluntad expresa de la Congregación del Sagrado Corazón para la participación de laicos en los vicerrectorados, fue continuada con otros profesores varones: Dr. Jorge Silva Merino y Dr. Fernando Elgegren Reátegui.

\section{Escuela de Posgrado}

- La inquietud por facilitar el ingreso de varones a la UNIFE, se centró en la Escuela de Posgrado considerando que los estudiantes ya son profesionales y no jóvenes como en el pregrado. La Hna. Elizabeth Bazán Gayoso, 
Q.D.D.G., Coordinadora del Programa Académico de Doctorado en Educación, planteaba que los estudios de posgrado ofrecian un espacio en donde la coeducación podría funcionar sin mayor dificultad. Esta inquietud la mantuvo presente durante un tiempo. Al convertirse en Rectora trajo a colación su interés académico por este tema.

- La Facultad de Derecho presenta una solicitud formal (Oficio No93-2015UNIFE-FD-D) para la admisión de varones a la maestría en la cual fundamenta razones de derecho de toda persona, la no discriminación por motivo de sexo y la igualdad jurídica del varón y de la mujer (Constitución de 1993, DS Nº 004-2008 y otras normas legales).

- La dirección de la Escuela de Posgrado encarga al Coordinador de la maestría de Derecho, Luis Felipe Bramont-Arias, el estudio jurídico el cual es elevado al rectorado junto con el oficio de la Escuela de Posgrado (Oficio No014/2015-EPG) de fecha 17/3/2015. En él se sugiere que la Congregación del Sagrado Corazón exprese su voluntad a dicha apertura y se tomen las acciones administrativas correspondientes para quelos diplomas de Gradodelos varones sean admitidos por la SUNEDU. Asimismo, se señala que, este interés surge desde hace ya muchos años. Debe mencionarse a la Hna. Gladys Buzzio Zamora, quien durante su rectorado impulsó reformas y planteó esta "apertura a varones comenzando por el Posgrado". Luego, el Consejo Universitario ha discutido la necesidad de estudiar el tema. Más aún, la Escuela de Posgrado, en otras oportunidades, planteó dicha posibilidad. La Escuela de Posgrado ha realizado consulta a los coordinadores (as) de los diferentes programas de Maestría y Doctorado quienes se muestran abiertos a esta iniciativa.

- Es así que el Consejo Universitario conforma una Comisión especial para hacer viable la propuesta conformada por tres de sus miembros: Victoria García García, Agustín Campos Arenas y Mario Romero Antola (CU No1193, 27/05/2015).

- La Hna. Carmen Durand Pardo, provincial de la Congregación, en respuesta a lo solicitado, se dirige al Consejo Universitario y señala la aprobación de la admisión de varones a los Programas Académicos de la Escuela de Posgrado y de las Segundas Especialidades (C.U. No 1197, 01/07/2015).

- El Consejo universitario, en consecuencia, aprueba el ingreso de varones a los estudios de Posgrado y Segundas Especialidades.

- En la admisión 2015-II, ingresan los tres primeros estudiantes varones al Programa de Maestría en educación: Docencia Universitaria.

- Miguel Ángel Díaz Sebastián

- Bruno Juan Díaz Sebastián

- Jonathan Peter Quiñonez Malpartida

En el año lectivo 2016 han ingresado 22 varones a los diferentes programas académicos distribuidos de la manera siguiente:

Tabla 2

Ingresantes varones a los programas académicos de la Escuela de Posgrado

\begin{tabular}{crccccc}
\hline Semestre & \multicolumn{2}{c}{ Educación } & Psicología & Derecho & Nutrición & \multirow{2}{*}{ TOTAL } \\
\cline { 2 - 6 } & Maestría & Doctorado & Maestría & Maestría & Maestría & \\
\hline $2016-I$ & 1 & 1 & 5 & $3(2)^{*}$ & - & $10(9)$ \\
2016-II & 5 & - & 1 & 4 & 2 & 12 \\
TOTAL & 6 & 1 & 6 & $7(6)$ & 2 & $22(21)$ \\
\hline
\end{tabular}

*Ingresaron 3, continuian 2 
O sea que actualmente 25 (24) [con los tres primeros ingresantes del 2015] varones están cursando estudios regulares en los Programas Académicos de la Escuela de Posgrado de la UNIFÉ.

\section{Respuestas a las preguntas del cuestionario}

Una pequeña encuesta se aplicó a los tres primeros varones ingresantes al Programa Académico de Maestría en Educación para conocer sus opiniones y experiencias ad portas de concluir sus estudios de maestría en UNIFÉ.

1. ¿Cómo se enteró que la Escuela de Posgrado (EPG) permitía el ingreso de varones a los programas de estudios?

- Fui informado en mi centro de trabajo:

- IPNM $(\mathrm{n}=2)$

- Departamento de Ciencias UNIFE $(n=1)$

2. ¿Cómo decidió venir a estudiar a la EPG? ¿Fue dificil?

- Fue fácil por la ubicación de mi centro laboral y por tener el horario adecuado.

- Decidi por motivos personales y profesionales, pues estaba en mis planes. Lo difícil fue tener el dinero.

- Por automotivación. Desde que laboro en la UNIFE quería estudiar una maestria en esta universidad. Fue fácil por los nuevos retos que pueden presentarse y lo más importante la actualización o crecimiento en los conocimientos.

3. ¿Cómo fueron las primeras semanas?

- Me adapté con facilidad porque los docentes y personal son muy amables.

- Me gustó mucho el nivel académico y la organización.
- Fue interesante por la calidad de docentes y la experiencia que tenian en los temas abordados.

4. A lo largo de estos tres ciclos de estudios, ¿cómo se ha sentido al ser de los primeros varones en la EPG?

- Me siento bien y con muchos deseos de obtener mi grado.

- Es un sentimiento que ya he tenido cuando estudie en el IPNM, me tocó ser el único varón en mi especialidad.

- Nunca me sentí mal en una universidad de mujeres ya que soy parte de la familia UNIFE.

5. ¿Cree Ud. que el proceso de coeducación en la EPG, es ya un hecho consumado?

- Está bastante avanzado

- En este momento estamos en proceso

- Se da en muchos cursos.

6. Otro (libre)

- Excelente, considerando nuestro contexto es adecuado que los grupos sean mixtos. Agradezco la oportunidad de estudiar en esta universidad.

- Hay un cambio en la didáctica por cuanto los estudiantes son personas profesionales.

\section{Conclusiones}

La universidad Femenina del Sagrado Corazón - UNIFÉ en Julio del 2015 aprueba el ingreso de varones a los Programas Académicos de la Escuela de Posgrado y Segundas Especialidades. Esto como resultado de un largo proceso que tienen sus origenes en la iniciativa del rectorado de la Hna. Gladys Buzzio Zamora, la decisión de la Hna. Elizabeth Bazán Gayoso (qdDg), rectora en el año 2015 y la aprobación expresa de la Congregación del Sagrado Corazón a través de 
la Hna Carmen Durand Pardo, provincial de la Congregación del Sagrado Corazón.

Hay tres pioneros que ingresaron a la Maestría en Educación, especialidad: Docencia Universitaria. Sus nombres quedaron señalados como el de los primeros varones en la Escuela de Posgrado de la UNIFÉ y que tuvieron según las respuestas al cuestionario, estudios satisfactorios en los tres semestres de estudios que contempla la Maestría.

Actualmente, hay 25 matriculados y 24 cursando estudios en todos los Programas Académicos de la EPG. El camino se ha iniciado y como dice el poeta "se hace camino al andar".

\section{Referencias}

Catholic Encyclopedia Coeducation: The practice of educating the sexes together. Recuperado de http:// //www.catholic.com/encyclopedia/ co-education

Collegestats.org (2015). What are America's first coed colleges?. Recuperado de https://www.cmc. edu/magazine/spring-summer-2015/whatare-americas-first-coed-colleges.

CQ Researcher (1969). Coeducation: New Growth. Recuperado de http://library. cqpress.com/cqresearcher/document. php?id=cqresrre 1969050700

Florida State University. About Florida State. Recuperado de https://www.fsu.edu/ about/history.html.

Gill, J. (2015). Changing from single sex to co-ed can be good if based on educational (not economic) reasons. Recuperado de http://www.aare.edu.au/ blog/?p=1126 (Julio 23).

Guest, M. (2014). Analysis and Research into coeducation in Australia and the Uk and the experience of these schools that change status. Recuperado de http://wwwaisnw. edu.au/Services/EducationResearch/ Latest $\% 20$ Research $\% 20$ Documents / Analysis $\% 20$ and $\% 20$ Research $\% 20$ into $\% 20$
Co-education $\% 20$ in $\% 20$ Australia $\% 20$ and $20 \%$ the $\% 20$ UK.pdf

Hicks, M. (2004). Integrating women at Oxford and Harvard Universities, 1964 - 1977. Recuperado de jitt's://dash.harvard.edu/bitstream/ handle / $1 / 4677608 / 35$ hicks $\% 20-\% 20$ with $\% 20$ changes.pages.pdf? sequence $=1$

Inside Higher Ed. (2013). Debates on Men at Women's College. Recuperado de https://www. insidehighered.com/news/2013/01/14/ debates-about-coeducation-wilson-andsalem-colleges

Online Courses, Staff Writers (2011) 15 fascinating effects about same-sex schools. Recuperado de http://www.onlinecollegecourses. com/2011/08/15-fascinating-facts-aboutsame-sex-schools/

Parmar, B. (2013). Coeducational school are bad for girls. Recuperado de http://www.theguardian. com/women-in-leadership/2013/oct/30/ co-educational-schools-bad-for-girls

Real Academia Española. Diccionario de la lengua española. Recuperado de http://www.dle.rae. es $/$ ?id $=$ DglqVCc

The National Bereau of Economic Research (2016). College Coeducation from 1835 to the present. Recuperado de http:www.nber.org/ digest/jan11/w16281.html

Thompson-Deveaux, A. (2016). When Women Came to Princeton. Recuperado de https:// paw.princeton.edu/article/when-womencame-princeton 